\title{
Micro Optical Viscosity Sensor for in situ Measurement Based on a Laser-Induced Capillary Wave"
}

\author{
Yoshihiro TAGUCHI $^{* *}$, Ryusuke NAGAMACHI ${ }^{* * *}$ and Yuji NAGASAKA ${ }^{* *}$ \\ ${ }^{* *}$ Department of System Design Engineering, Keio University, \\ 3-14-1 Hiyoshi, Yokohama 223-8522, Japan \\ E-mail: tag@sd.keio.ac.jp \\ ***School of Integrated Design Engineering, Keio University, \\ 3-14-1 Hiyoshi, Yokohama 223-8522, Japan
}

\begin{abstract}
In this article, we demonstrate a novel micro optical viscosity sensor (MOVS) based on a laser-induced capillary wave with a focus control system that enables in situ monitoring of viscosity and surface tension changes in microliter-order liquid samples such as body fluids, polymer coating materials, lubricants, heavy oils and so on. The microfabricated sensor consists of two deep trenches (depth of $273 \mu \mathrm{m}$ ) holding photonic crystal fibers (PCFs), and three shallow trenches (depth of 125 $\mu \mathrm{m})$ holding collimating lensed fibers (CLFs) for the probing laser. The capillary wave is excited by two pulsed laser beams generating optical interference, and the rapid motion of the capillary wave, which contains information regarding the viscosity and surface tension of the sample, is monitored by detecting the first-order diffracted beam of the probing laser irradiated onto the sample surface. In order to apply this sensor in manufacturing and clinical settings, the distance between the liquid level and the sensor must be properly adjusted because the sample surface is strongly influenced by evaporation and outside vibration disturbances under such conditions. In the MOVS, the specular reflection of the probing laser is detected by a symmetrically placed collimating lensed fiber. Maximizing the signal of specular reflection by using a piezo stage connected to the MOVS and PID controller, the focal points of the fibers (PCFs and CLFs) are adjusted on the sample surface. The high-reproducible measurement results under evaporation and outside disturbance indicate the validity of MOVS for in situ application.
\end{abstract}

Key words: in-situ Monitoring, Laser-Induced Capillary Wave, Optical Interference, Optical Viscometer Chip, Surface Tension, Viscosity

\section{Introduction}

The viscosity of a liquid is an essential physical property in numerous industrial fields (e.g. the polymer industry ${ }^{(1)}$, the petroleum industry ${ }^{(2)}$, the food industry ${ }^{(3)}$, and so on) and material science for thermal design of liquid flow and its system. For example, in the polymer industry, real-time monitoring and in situ scanning of viscosity changes due to the formation and modification of polymers enables process control of polymerization, which can enhance the quality of products as well as the productivity of new polymer materials. In contrast, in the medical field, on-site monitoring of viscosity provides a fast and relatively noninvasive means of measuring small sample volumes of bodily fluids such as blood to provide information related to circulatory system disease (e.g. thrombosis ${ }^{(4)}$ ). This technique could potentially reduce the treatment burden for patients and thus improve their 
quality of life (QOL). Furthermore, in this area of research and development, the massively parallel sensing of different materials, so-called "combinatorial synthesis ${ }^{(5) ",}$ allows to promote the advanced research and development of material. However, the conventional viscosity measurement techniques (e.g. the capillary method, the falling-body method, and the vibrating method ${ }^{(6,7)}$, well known as standard bulk methods, require a large sample volume (a few tens of milliliters) and a long measurement time (a few minutes), and thus these techniques cannot fundamentally suit the demands mentioned above.

Several groups have developed miniaturized viscometers using microelectromechanical systems (MEMS) that can satisfy some of the above needs. For example, cantilever-type micro viscometric devices have been monolithically fabricated on a single-crystal silicon wafer $^{(8-10)}$. The resonant frequency of an oscillated cantilever provides the viscosity of a micro-liter order sample in which the device is immersed. Zhao et al ${ }^{(10)}$ have demonstrated a MEMS viscometric glucose sensor for monitoring glucose levels in diabetics. For the measurements carried out over a broad range of viscosities, temperatures, and shear rates, micro channel/capillary viscometers have been proposed ${ }^{(11,12)}$. Chevalier et $\mathrm{al}^{(12)}$ have measured the viscosities of oil- and ethanol-based nanofluids up to $10^{-5} \mathrm{~s}^{-1}$ using micromachined capillaries on chip rheometers. Despite the considerable amount of research in this field, a non-contact viscosity sensor applicable to in situ, as depo or in process measurement where the viscosity changes rapidly, has not been proposed, because almost all of the devices are required to come in contact with the sample, thus introducing a risk of contamination.

We have developed a novel micro optical viscosity sensor (MOVS) based on a laser-induced capillary wave that enables in situ real-time monitoring of viscosity and surface tension change in micro-liter order liquids. The signal change due to the viscosity change has been successfully observed ${ }^{(13)}$. In order to apply this sensor in manufacturing and clinical settings, the distance between the liquid level and the sensor needs to be adjusted appropriately. In the present study, the applicability of MOVS with a focus control system for in situ monitoring was investigated using liquid samples, which are strongly influenced by evaporation and outside vibrational disturbances.

\section{Nomenclature}

$\begin{array}{cl}C_{\mathrm{p}} & : \text { specific heat capacity, } \mathrm{kJ} / \mathrm{kg} \mathrm{K} \\ I_{\mathrm{h}} & : \text { pulsed heating laser energy, } \mathrm{J} / \mathrm{m}^{2} \\ T_{0} & : \text { mean of temperature distribution, } \mathrm{K} \\ T_{1} & : \text { amplitude of temperature distribution, } \mathrm{K} \\ u_{0} & : \text { spatially uniform displacement, } \mathrm{m} \\ u_{1} & : \text { amplitude of the laser-induced capillary wave, } \mathrm{m} \\ V L & : \text { sound velocity of liquid, } \mathrm{m} / \mathrm{s} \\ V i & : \text { visibility of interference fringe, - } \\ V i_{\mathrm{BP}} & : \text { visibility detected by the beam profiler, - } \\ \Delta z_{\mathrm{d}} & : \text { focal depth, m }\end{array}$

\section{Greek}

$\begin{array}{cl}\alpha & : \text { absorption coefficient, } 1 / \mathrm{m} \\ \beta & : \text { thermal expansion coefficient, } 1 / \mathrm{K} \\ \Lambda & : \text { interference fringe space, } \mathrm{m} \\ \delta_{\mathrm{BP}} & : \text { half-pixel size of the beam profiler, } \mathrm{m} \\ \phi & : \text { incident angle of probing beam, deg } \\ \eta & : \text { viscosity, Pa } \mathrm{s} \\ \lambda & : \text { thermal conductivity, } \mathrm{W} / \mathrm{m} \mathrm{K} \\ \lambda_{\mathrm{h}} & : \text { wavelength of heating laser, } \mathrm{m}\end{array}$


$\lambda_{\mathrm{p}} \quad$ : wavelength of probing laser, $\mathrm{m}$

$\theta \quad$ : incident angle of heating beam, deg

$\rho \quad:$ density, $\mathrm{kg} / \mathrm{m}^{3}$

\section{Measurement Principle}

\subsection{Laser-induced Capillary Wave Method}

A schematic of the measurement principle of MOVS is shown in Fig. 1. The laser-induced capillary wave method was utilized for the optical viscometry. Terazima et $\mathrm{al}^{(14)}$ first demonstrated the laser-induced capillary wave method for the investigation of surface interactions of a gas/liquid interface, and Nagasaka et $\mathrm{al}^{(15)}$ have developed a bench-top-type in situ viscometer using a nano-second pulsed carbon dioxide laser for the control of industrial materials such as food. The sample surface is heated by two pulsed laser beams, which intersect and generate an optical interference fringe pattern on the surface. The optical intensity distribution $I(x)$ on the surface can be written as,

$$
\begin{aligned}
& I(x)=I_{\mathrm{h}}\left\{1+\cos \left(\frac{2 \pi x}{\Lambda}\right)\right\}, \\
& \Lambda=\frac{\lambda_{\mathrm{h}}}{2 \sin (\theta / 2)},
\end{aligned}
$$

where $I_{\mathrm{h}}$ is the intensity of the heating laser, $\Lambda$ is the fringe space, $\lambda_{\mathrm{h}}$ is the wavelength of the excitation laser, and $\theta$ is the beam intersecting angle. The sample surface is instantaneously heated by optical interference, and the temperature distribution $T(x)$ is then expressed by the following expression.

$$
T(x)=T_{0}+T_{1} \cos \left(\frac{2 \pi x}{\Lambda}\right),
$$

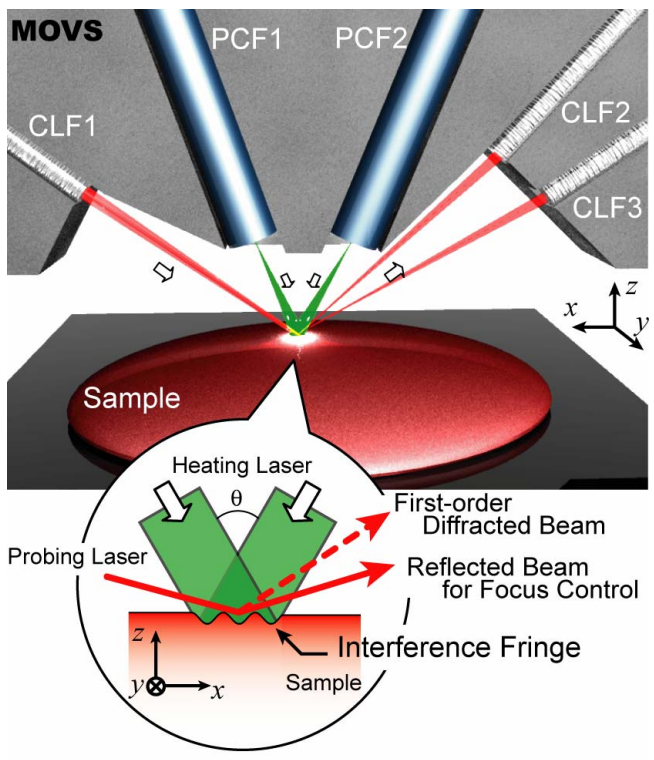

Fig. 1 Measurement principle of a micro optical viscosity sensor (MOVS). The sample surface is heated by the interference pattern of pulsed laser beams that are delivered through photonic crystal fibers (PCFs). The probing laser beam is irradiated onto the laser-induced capillary wave, and the first-order diffracted beam and specular reflected beam are detected via collimating lensed fibers (CLFs). 
where $T_{0}$ and $T_{1}$ are the mean and the amplitude of the temperature distribution, respectively. The spatially periodic temperature distribution generates a capillary wave (nanometer order of amplitude, and micro-meter order of wavelength) due to thermal expansion and the temperature dependence of the sample's surface tension. The surface displacement $u(x)$ due to the capillary wave is expressed as,

$$
u(x)=u_{0}+u_{1} \cos \left(\frac{2 \pi x}{\Lambda}\right)
$$

where $u_{0}$ is the spatially uniform displacement and $u_{1}$ is the amplitude of the laser-induced capillary wave. The geometric behavior of the laser-induced capillary wave is described by three phenomenological equations with the appropriate boundary conditions. First, the continuity equation is written as,

$$
\frac{\partial \rho}{\partial \mathrm{t}}+\nabla \cdot(\rho \mathbf{V})=0
$$

where $\rho$ is the density and $\mathbf{V}$ is the velocity of the liquid. Second, the Navier-Stokes equation is expressed by,

$$
\frac{\partial^{2} \mathbf{u}}{\partial t^{2}}-v \nabla^{2} \frac{\partial \mathbf{u}}{\partial t}-V_{\mathrm{L}}^{2} \nabla \nabla \cdot \mathbf{u}=-\beta V_{\mathrm{L}}^{2} \nabla T,
$$

where $\mathbf{u}$ is the displacement, $\beta$ is the thermal expansion coefficient, $V_{\mathrm{L}}$ is the sound velocity in the liquid, and $v=\eta / \rho$ is the kinematic viscosity as a constant of viscosity $\eta$ divided by the density $\rho$. Third, the heat conduction equation is given by the following expression,

$$
\rho C_{\mathrm{p}}\left(\frac{\partial T}{\partial t}\right)-\lambda \nabla^{2} T=I_{\mathrm{h}} \alpha\left\{1+\cos \left(\frac{2 \pi x}{\Lambda}\right)\right\} \exp (\alpha z) \delta(t)
$$

where $C_{\mathrm{p}}$ is the heat capacity, $\lambda$ is the thermal conductivity, and $\alpha$ is the absorption coefficient of light. The boundary conditions are expressed as follows,

$$
\begin{aligned}
& \sigma \frac{\partial^{2} u_{x}}{\partial x^{2}}-2 \frac{\partial}{\partial t}\left(\frac{\partial u_{z}}{\partial z}\right)+\rho V_{\mathrm{L}}^{2}\left(\alpha T-\frac{\partial u_{x}}{\partial x}-\frac{\partial u_{z}}{\partial z}\right)=0 \\
& \eta \frac{\partial}{\partial t}\left(\frac{\partial u_{x}}{\partial z}+\frac{\partial u_{z}}{\partial x}\right)-\left(\frac{\partial \sigma}{\partial T}\right) \frac{\partial T}{\partial x}=0 .
\end{aligned}
$$

$\sigma$ denotes the surface tension, and the temperature dependence of the surface tension that is sinusoidally distributed on the sample surface is considered in the second term of Eq. (9). The amplitude of the laser-induced capillary wave $u_{1}$ can be derived from the above expressions as a function of the physical properties of the liquid and the experimental condition written in Laplace domain. The detail of the Laplace transformed amplitude is written in the reference ${ }^{(16)}$. Consequently, by observing the motion of the laser-induced capillary wave, the viscosity in the near surface and surface tension are numerically analyzed from the experimental data of the damped oscillating signal by inverse problem analysis. In order to measure the geometric behavior in the relaxation process of the laser-induced capillary wave after pulsed heating, which contains the information regarding viscosity and surface tension, a probing beam is irradiated onto the laser-induced capillary 
wave, and the first-order diffracted beam is detected since the laser-induced capillary wave acts as a dynamic reflection grating. The intensity of the first-order diffracted beam $I_{\text {sig }}(t)$ is proportional to the square of the amplitude of the laser-induced capillary wave.

$$
I_{\text {sig }}(t) \propto u_{1}^{2}(t) .
$$

Hence, by detecting the first-order diffracted beam, the dumping oscillation behavior of the laser-induced capillary wave can be observed. In the MOVS, all laser beams are delivered, irradiated and collected by photonic crystal fibers (PCFs) and collimating lensed fibers (CLFs) whose focal points and optical axes intersect with each other.

\subsection{Focus Control Principle}

For the in situ measurement of viscosity, the focus control system is required to reduce the negative effects of liquid level reduction and fluctuations due to evaporation of the sample and external vibrations, which are dominant factors in the control of the production process such as polymer coating process. In this research, specular reflection of the probing beam was utilized as a control signal of liquid levels. The probing beam delivered via CLF1 is irradiated onto the sample surface, and the first-order diffracted beam is detected through CLF2 as the signal of the laser-induced capillary wave, as previously mentioned in section 2.1. Meanwhile, the specular reflection of the probing beam is detected by CLF3 as the reference signal for the focus control feedback. The dual-axes configuration consisting of two CLFs is shown in Fig. 2. The point spread function (PSF) of the dual-axes configuration is the product of individual PSFs of CLF1 and CLF3, and the focal depth $\Delta z_{\mathrm{d}}$ is described as ${ }^{(17)}$,

$$
\Delta z_{\mathrm{d}}=\frac{0.37 \lambda_{\mathrm{p}}}{\mathrm{NAsin} \phi}
$$

where $\lambda_{\mathrm{p}}$ is the wavelength of the probing beam, NA is the numerical aperture of the CLFs, and $\phi$ is the incident angle of the probing beam of CLF1 and the detection port of CLF3. The focal depth of MOVS is estimated to be $12.6 \mu \mathrm{m}$ by Eq. (11) in the case of $\phi=60^{\circ}$. Maximizing the signal of the specular reflection by using a PID controller and a piezo stage (PZT, cutoff frequency of $600 \mathrm{~Hz}$ ) connecting to the MOVS chip, the focal points of CLFs and the intersecting points of PCFs are adjusted on the sample surface.

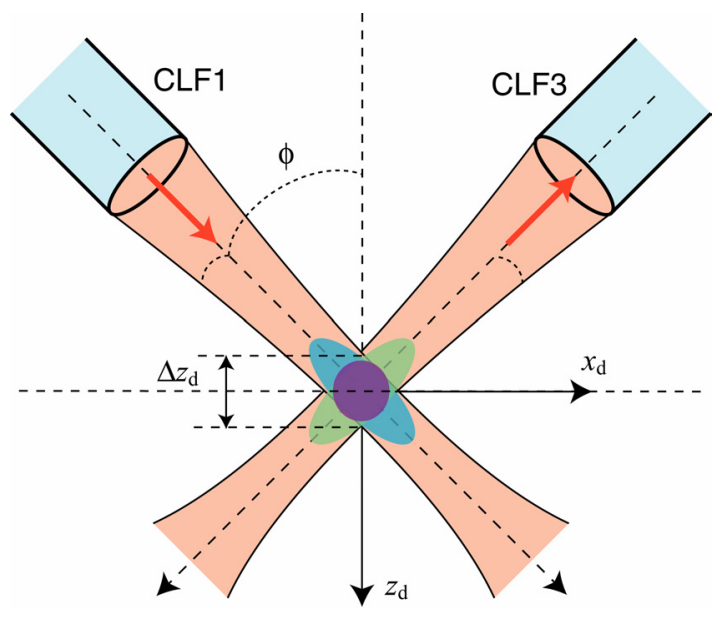

Fig. 2 Schematic diagram of the dual-axes configuration. 


\section{Device Configuration and Experimental Setup}

\subsection{Design and Fabrication of the Micro Optical Viscosity Sensor (MOVS)}

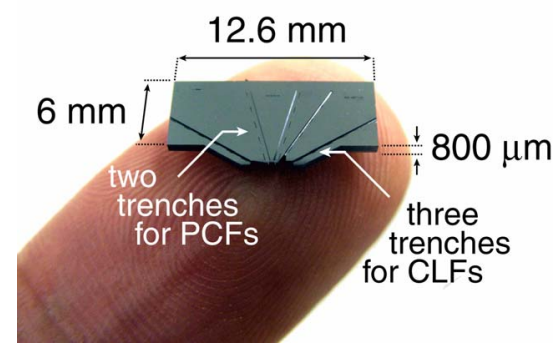

Fig. 3 Overall view of MOVS consisting of two wide and deep trenches holding PCFs and three narrow and shallow trenches holding CLFs.

Figure 3 shows an overall view of the MOVS without fibers. The MOVS (chip size of $12.6 \mathrm{~mm} \times 6 \mathrm{~mm} \times 800 \mu \mathrm{m})$ consists of two wide and deep trenches for the fibers of the excitation laser placed in the center at an intersecting angle of $\theta$ corresponding to the fringe space of interference, and three narrow and shallow trenches for the fibers of the irradiating and collecting probing laser placed in the side of the center trenches at an angle $\phi$ and the first-order diffraction angle $\phi_{1}$ that is described as,

$$
\phi_{1}=\sin ^{-1}\left(\lambda_{\mathrm{p}} / \Lambda-\sin \phi\right) \text {. }
$$

The five trenches with springs can precisely hold the fibers within $\pm 1 \mu \mathrm{m}$ because the MOVS chip is fabricated using MEMS technology. The PCFs (core diameter of $25.2 \mu \mathrm{m}$, mode-field diameter of $19.8 \mu \mathrm{m}$, ultra small NA of 0.04 at a wavelength of $1064 \mathrm{~nm}$, clad diameter of $268 \mu \mathrm{m}$ ) can deliver the high-power short pulsed laser beam onto the sample surface to generate the laser-induced capillary wave. For the probing and detecting fibers, collimated lensed fibers are utilized in which the index-grating multi-mode fibers (clad diameter of $125 \mu \mathrm{m}$, length of $935 \mu \mathrm{m}$, and the spot size of $16.7 \mu \mathrm{m}$ at the working distance of $3 \mathrm{~mm}$ ) are fusion-spliced with single mode fibers.

The diameter of the PCF is approximately twice the size of the CLF; therefore, depth control of the CLF is required in the fabrication process. The MOVS is fabricated on a silicon-on-insulator (SOI) wafer by timed-etch deep reactive ion etching (DRIE) with an inductively coupled plasma (ICP) creating the fiber grooves, springs for holding the fibers, the dummy trenches, and the alignment edge for the fiber end face, according to a process flow schematically drawn in Fig. 4. First, a thermal oxide layer (thickness of $400 \mathrm{~nm}$ ) and an aluminum layer of $300 \mathrm{~nm}$ are grown and sputtered on the SOI wafer (the device layer of $269 \mu \mathrm{m}$, the buried oxide layer of $4 \mu \mathrm{m}$, and the substrate of $525 \mu \mathrm{m}$ ) as photomask layers, as shown in Fig. 4(a). In order to form the grooves on the SOI wafer for the PCFs and CLFs that lead the heating and probing lasers, the photomask layers are patterned by a photolithography technique followed by etching of the layers using phosphoric acid aluminum etchant and reactive ion etching (RIE). The 8- $\mu \mathrm{m}$ photoresist is spin-coated and patterned to obtain the mask for the timed-etch DRIE process in order for the center of the fiber cores of the PCF and CLF to be aligned at the same height (Fig. 4(b)). Because the aspect ratio dependent etching (ARDE) effect and the micro loading effect in the grooves may occur in the case that wide and narrow trenches are patterned in the same wafer, the dummy trenches are patterned in the grooves of the PCF (Fig. 4(c)). The first and second etches were carried out by using timed-etch DRIE until the depth of the trenches for the CLFs reached the appropriate position (Fig. 4(d)). For the back-side etching, which shaped the outline of the tip of MOVS in order to control the working distance of the chip, a silicon 
dummy wafer was bonded to the foreside of the processed wafer by photoresist, and the two windows were etched to access the foreside alignment mark for the backside alignment (Fig. 4(e)). After backside etching, the dummy trenches and springs were released by etching the sacrificial oxide layer using buffered hydrofluoric acid, and finally the fibers were packaged in the trenches by a micromanipulator (Fig. 4(f-h)).

A scanning electron microscope image of the MOVS in which the fibers are packaged and glued by UV resin is shown in Fig. 5. Although ion bombardment of the sidewall and the "mousebite effect" occurred to some extent during DRIE process, undercutting was not observed in the fabricated structures. Therefore, the trenches of the PCFs and CLFs can precisely hold the fibers with springs, and the centers of the two different fibers are aligned at the same height.

a)

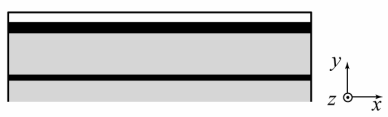

b)

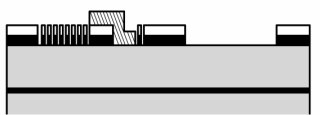

c)

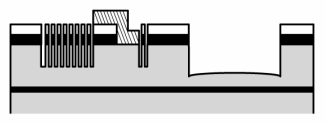

d)

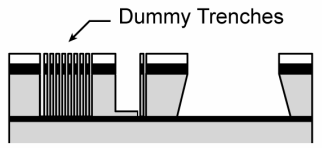

e)
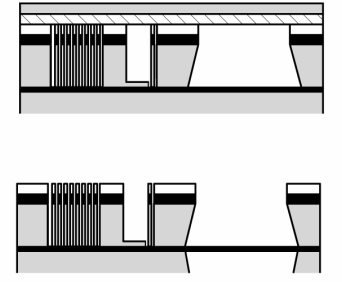

g)

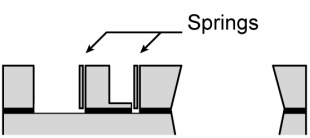

h)

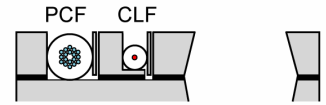

Silicon

Silicon dioxide Aluminum

Photoresist

Fig. 4 Process flow of the fabrication of the MOVS

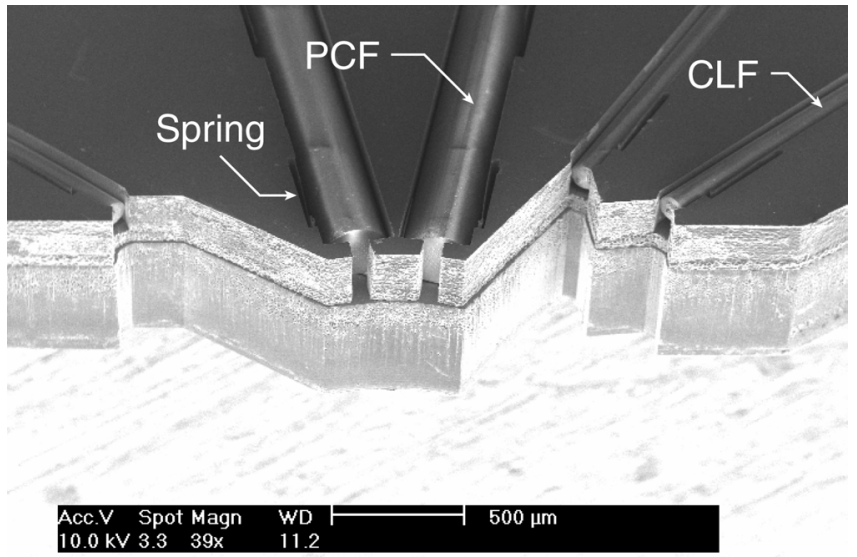

Fig. 5 A scanning electron microscope (SEM) image of microfabricated MOVS.

\subsection{Measurement Apparatus}

Figure 6 shows a schematic diagram of the optical system of a MOVS. We utilize a $\mathrm{Nd}$ :YAG laser (wavelength of $1064 \mathrm{~nm}$, power of $3 \mathrm{~mJ}$, and a pulse width of $6 \mathrm{~ns}$ ) to generate the capillary wave on the sample surface. The pulsed laser beam is first shaped by lenses 1 and 2 (L1 and L2) to achieve an optimal beam diameter, and then divided into two beams of equal intensity by a nonpolarized beam splitter (NPBS). The two beams are coupled into PCFs by using the objective lens (OBJ) with a single-mode fiber coupler, and are then intersected on the sample surface, generating the interference fringe pattern. The sample surface is interferometrically excited, and the laser-induced capillary wave is generated. The probing laser beam (wavelength of $641 \mathrm{~nm}$, and maximum power of 20 
$\mathrm{mW}$ ) is led to the sample surface via CLF1. The first-order diffracted beam is collected by CLF2, and is detected by a cooled photomultiplier tube (PMT) with a low-noise high-speed current amplifier. The specular reflection of the probing beam for the focus control signal of the MOVS chip is simultaneously detected by a Si-PIN photodetector (PD). The cutoff frequency of the photodetector (up to $200 \mathrm{kHz}$ ) is much lower than that of the dumping oscillation of the laser-induced capillary wave, and the intensity of the first-order diffracted beam is significantly lower than that of the specular reflection; the diffraction beam can therefore be assumed to not affect the focal depth control signal.

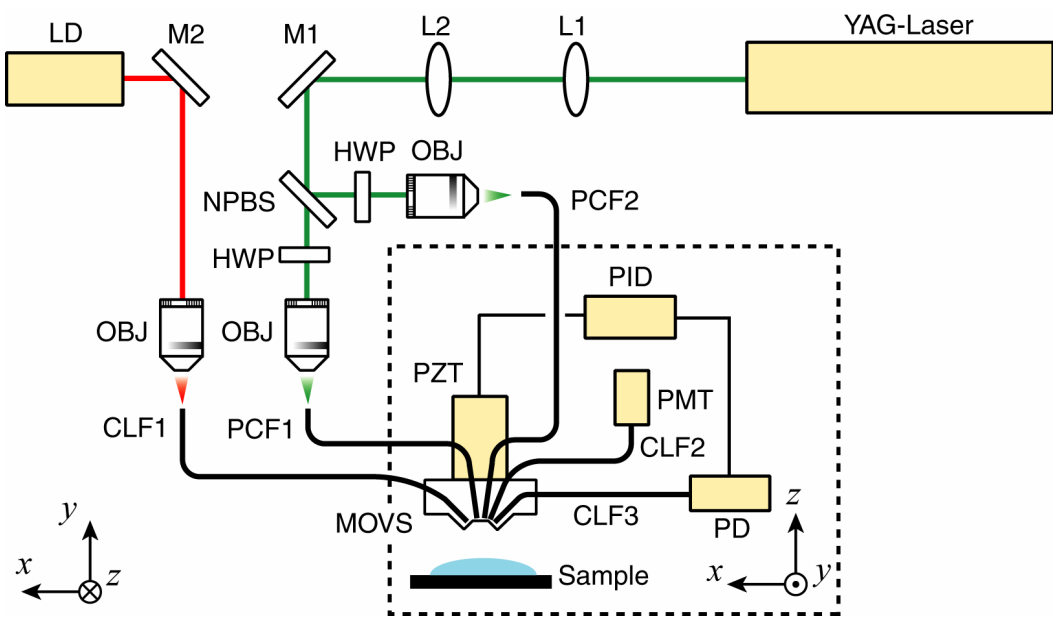

Fig. 6 Schematic of the optical system of the MOVS.

\section{Results and Discussion}

In order to verify the feasibility of the presented MOVS, a point spread function (PSF) was performed in the $z$-direction, and the interference fringe of the excitation laser delivered by PCFs was observed, as shown in Figs.7 and 8. Figure 7 indicates the optical response of the specular reflection during the $z$-axis scanning of distilled water. The signal at the out-of-focus position $(\mathrm{z}= \pm 5 \mu \mathrm{m})$ was significantly fluctuated, because the specular reflection signal in this region was sensitive to small variations in liquid level. The full-width of half-maximum of PSF was estimated to be $10.7 \mu \mathrm{m}$, which agreed with the theoretical value calculated in section 2.2. The deviation was caused by the uncertainty of positioning, which could be changed due to outside disturbance. The two short-pulsed beams successfully formed an interference pattern at a cross section of the two beams as shown in Fig. 8. The interference fringe was observed by a microscopic beam profiler (pixel

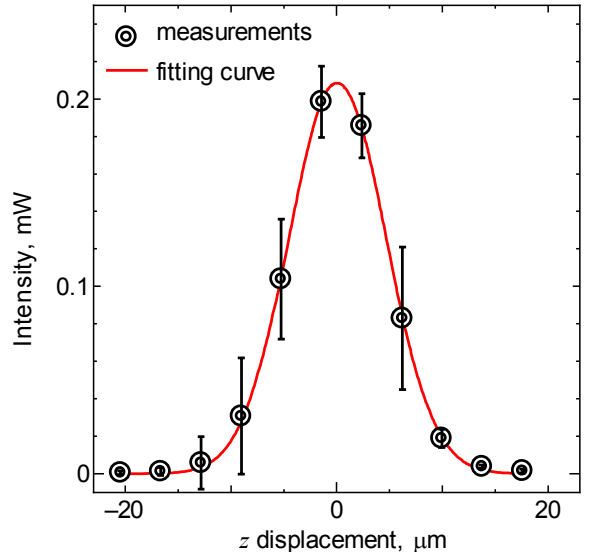

Fig. 7 PSF of dual-axes configuration of CLFs.

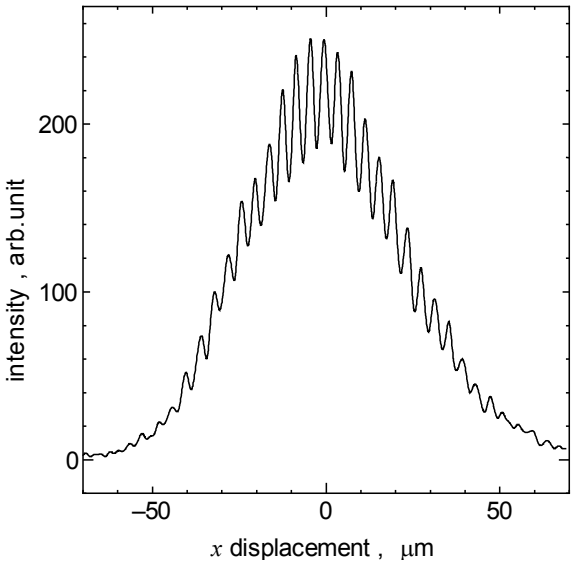

Fig. 8 Interference fringe generated by heating laser. 
size of $4.65 \mu \mathrm{m}$, objective lens of fourfold magnification), and the fringe space was estimated to be $3.96 \mu \mathrm{m}$, which was in agreement with the designed value of $4 \mu \mathrm{m}$. The visibility of the interference fringe was calculated as 0.14 . In fact, the pixel size of the beam profiler is close to the fringe space; therefore the image captured by the beam profiler is averaged. The actual visibility $V i$ is described by the following expression,

$$
V i=\frac{\Lambda}{2 \pi \delta_{\mathrm{BP}}} V i_{\mathrm{BP}} \sin \left(\frac{2 \pi \delta_{\mathrm{BP}}}{\Lambda}\right),
$$

where $\delta_{\mathrm{BP}}$ is half the size of the pixel, and $V i_{\mathrm{BP}}$ is the visibility calculated from the image of the beam profiler. In the present setup, the actual visibility $V i$ is estimated as 0.17 , which is quite low due to the optical pass difference between the two pulsed laser beams. The coherent length of the pulsed YAG laser is around a few centimeters, thus there is still room for improvement in the visibility by adjusting the fiber length, which directly corresponds to the intensity of the first-order diffracted beam.

The specular reflected light signal of the probing beam and the displacement of the piezo stage are shown in Fig. 9. The MOVS tracked the reduction of liquid levels due to evaporation of the sample (Fig. 9(a)). The CLF1 was focused on the sample surface for a prolonged measurement period, even if the sample had evaporated; therefore, the fluctuation of the signal under control was dramatically decreased by using the control system. The deviation was calculated to be approximately $\pm 0.7 \mu \mathrm{m}$. Finally, to assess the validity of the focus control system for the in situ measurement of viscosity, we demonstrated the stability and reproducibility of the first-order diffracted beam of the

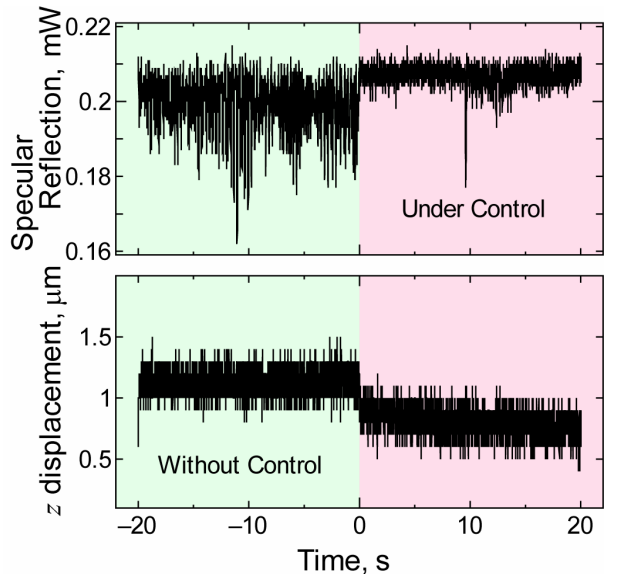

(a) evaporation

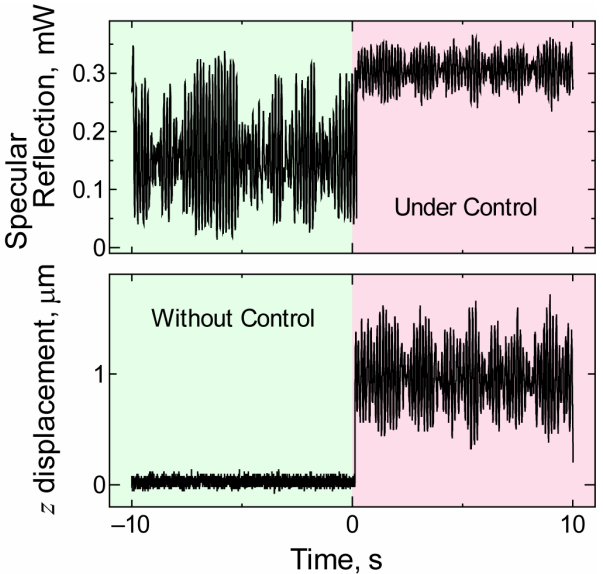

(b) outside vibration

Fig. 9 Focus control of MOVS against evaporation and vibration.

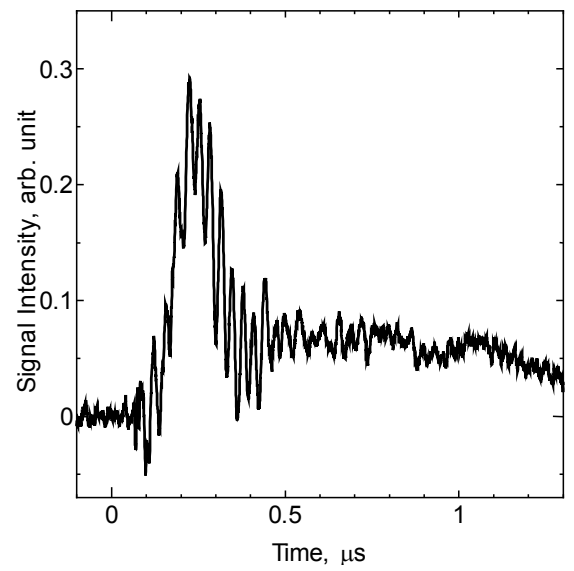

(a) without control

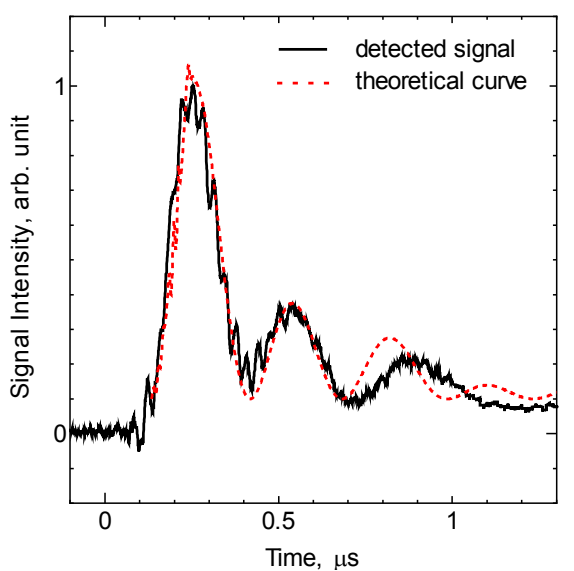

(b) under control

Fig. 10 First-order diffracted beam of laser-induced capillary wave of distilled water with pigment. 
laser-induced capillary wave using a mechanically vibrated sample of distilled water with $0.01 \%$ pigment (black carbon) at room temperature and atmospheric pressure. The oscillation (amplitude of $13 \mu \mathrm{m}$ and frequency of up to $7 \mathrm{~Hz}$ ) was applied to the sample, and the specular reflection was monitored, as shown in Fig. 9(b). The piezo stage was actuated to maintain the specular reflected signal against the microvibrational disturbance. The standard deviation without control was evaluated to be $30 \mu \mathrm{W}$; in contrast, the standard deviation under control was improved threefold (standard deviation of $10 \mu \mathrm{W}$ ). In Fig. 10, the first-order diffracted beams were averaged 256 times for 2 min while the vibration was applied. The signal-to-noise ratio (SNR) of the controlled laser-induced capillary wave signal ( $\mathrm{SNR}=250$ ) is dramatically improved over the uncontrolled signal ( $\mathrm{SNR}=66$, see Fig. (10(a)), and the high-speed damping oscillation (especially 2 nd peak and 3rd peak of oscillation) within $1 \mu$ s was clearly observed, as shown in Fig. 10(b). The detected signal under control was highly reproducible and in good agreement with the theoretical curve. By analyzing the damping oscillation signal, the kinematic viscosity was estimated to be 0.61 $\mathrm{mm}^{2} / \mathrm{s}$. Although there was an effect of dispersing media (low concentration of pigment), the estimated value was valuable compared with the reference value of the water ${ }^{(18)}$. Consequently, the applicability of the MOVS for non-contact and disturbance-free viscometry, which was promising in situ measurement where the viscosity was drastically changed in short time such as polymer coating, was verified.

\section{Conclusion}

A novel micro optical viscosity sensor (MOVS) with a focus control system has been proposed for in situ applications. The laser-induced capillary wave was successfully generated on the sample surface where the evaporation and ambient vibration strongly affected the liquid level, and the highly reproducible damped oscillation signal of the laser-induced capillary wave was detected using a MOVS with a focus control system. The validity of a MOVS that can track sample evaporation within $1 \mu \mathrm{m}$ and compensate for ambient vibrations, was confirmed. The measurement results using distilled water with $0.01 \%$ pigment indicate the applicability of MOVS for in situ measurement.

\section{Acknowledgements}

We gratefully appreciate Mr. Hiroshi Abe for his generous help in fabrication of the MOVS chip. The microfabrication of MOVS was conducted at the Center for Nano Lithography \& Analysis, The University of Tokyo, and the Nano Processing Partnership Platform, AIST. This research was partially supported by the Japan Society for the Promotion of Science (JSPS), Grant-in-Aid for Scientific Research (S, No.19206004), and Japan Science and Technology Agency (JST), Project to develop "Innovative Seeds".

\section{References}

(1) Tjong, S.C., Structure, Morphology, Mechanical and Thermal Characteristics of the in situ Composites based on Liquid Crystalline Polymers and Thermoplastics, Materials Science and Engineering R: Reports, Vol. 41, No. 1-2 (2003), pp. 1-60.

(2) Miller, K.A., Nelson, L.A. and Almond, R.M., Should You trust Your Heavy Oil Viscosity Measurement?, Journal of Canadian Petroleum Technology, Vol. 45, No. 4 (2006), pp. 42-48.

(3) Borne, M., Food Texture and Viscosity -Concept and Measurement 2nd edn, (2002), Academic.

(4) Dintenfass, L., Blood Microrheorogy, (1971), Butterworth.

(5) Webster, D.C., Radical Change in Research and Development: The Shift from Conventional Methods to High Throughput Methods, Journal of Coatings Technology, Vol. 2, No. 15 (2005), pp. 24-29. 
(6) Wakeham, W.A., Nagashima, A. and Sengers, J.V., Measurement of the Transport Properties of Fluids, Experimental Thermodynamics, Vol. III, (1991), Blackwell.

(7) The Japan Society of Mechanical Engineers, Thermophysical Properties Measurement Methods, Their Development and Engineering Applications, (1991), Yokendo, (in Japanese).

(8) Ronaldson, K.A., Fitt, A.D., Goodwin, A.R.H. and Wakeham, W.A., Transversely Oscillating MEMS Viscometer: The "Spider", International Journal of Thermophysics, Vol. 27, No. 6 (2006), pp. 1677-1695.

(9) Goodwin, A.R.H., Fitt, A.D., Ronaldson, K.A. and Wakeham, W.A., A Vibrating Plate Fabricated by the Methods of Microelectromechanical Systems (MEMS) for the Simultaneous Measurement of Density and Viscosity: Results for Argon at Temperatures Between 323 and $423 \mathrm{~K}$ at Pressures up to $68 \mathrm{MPa}$, International Journal of Thermophysics, VO1. 27, No. 6 (2006), pp. 1650-1676.

(10) Zhao, Y.J., Davidson, A., Bain, J., Li, S.Q., Wang, Q. and Lin Q, A MEMS Viscometric Glucose Monitoring Device, Proceedings of te 13th International Conference on Solid-State Sensors, Actuators and Microsystems, (2005-6), pp. 1816-1819.

(11) Moon, D., Bur, A.J. and Migler, K.B., Multi-sample Micro-slit Rheometry, Journal of Rheology, Vol. 52, No. 5 (2008), pp. 1131-1142.

(12) Chevalier, J. and Ayela, F., Microfluidic on Chip Viscometers, Review of Scientific Instruments, Vol. 79, No. 7 (2008), pp. 0761021-0761023.

(13) Taguchi, Y., Ebisui, A. and Nagasaka, Y., Miniaturized Optical Viscosity Sensor based on a Laser-induced Capillary Wave, Journal of Optics A: Pure and Applied Optics, Vol. 10, No. 4 (2008), pp. 0440081-6.

(14) Yasumoto, K., Hirota, N. and Terazima, M., Laser-induced Capillary Wave at Air/Liquid Interfaces in Time Domain, Applied Physics Letters, Vol. 74, No. 10 (1999), pp. 1495-1497.

(15) Oba, T, Kido, Y. and Nagasaka, Y., Development of Laser-induced Capillary Wave Method for Viscosity Measurement using Pulsed Carbon Dioxide Laser, International Journal of Thermophysics, Vol. 25, No. 5 (2004), pp. 1461-1473.

(16) Iwashima, H., Yabui, K. and Nagasaka, Y., Viscosity and Surface Tension Measurement by Laser-induced Capillary Wave Method (Development of Technique for Solving Inverse Problem), Transactions of the Japan Society of Mechanical Engineers, Series B, Vol. 73, No. 733 (2007), pp. 114-120.

(17) Corle, T.R. and Kino, G.S., Confocal Scanning Optical Microscopy and Related Imaging Systems, (1996), Academic Press.

(18) The Japan Society of Thermophysical Properties, Thermophysical Properties Handbook, (2008), Yokendo, (in Japanese). 